\title{
Transfer matrices for magnetized CMB anisotropies
}

\author{
Massimo Giovannini* \\ Centro “Enrico Fermi”, Compendio del Viminale, Via Panisperna 89/A, 00184 Rome, Italy, \\ and Department of Physics, Theory Division, CERN, 1211 Geneva 23, Switzerland
}

(Received 2 April 2006; published 17 May 2006)

\begin{abstract}
Large-scale magnetic fields can affect scalar cosmological perturbations whose evolution is described in the conformally Newtonian gauge and within the tight coupling approximation. The magnetized curvature perturbations present after matter-radiation equality (and prior to decoupling) are computed in terms of an appropriate transfer matrix allowing a general estimate of the Sachs-Wolfe plateau. From the observation that CMB initial conditions should be (predominantly) adiabatic, the contribution of the magnetic field intensity can be constrained.
\end{abstract}

DOI: 10.1103/PhysRevD.73.101302

Large-scale magnetic fields are observed at a $\mu \mathrm{G}$ level in galaxies, clusters and in some superclusters [1]. Compressional amplification (taking place during the gravitational collapse of the protogalaxy) allows to connect the observed magnetic field to a protogalactic field, present prior to gravitational collapse, of typical strength of $0.1 \mathrm{nG}$. A better understanding of the interplay between dynamo theory and the global conservation laws of magnetized plasmas has been recently achieved [2] also because of the improved comprehension of the solar dynamo action. It is then plausible, within the dynamo hypothesis, that the protogalactic field could be even much smaller than the $\mathrm{nG}$ and still explain some crucial properties of our magnetized Universe.

Thanks to magnetic flux (and magnetic helicity) conservation in a conductive plasma, a magnetic field of $\mathrm{nG}$ strength at the epoch of galaxy formation can be as large as $\mathrm{mG}$ (i.e. roughly 6 orders of magnitude larger) at the epoch of photon decoupling, i.e. for $z_{\mathrm{dec}}=1100$. If largescale magnetic fields have primordial origin, they were present prior to matter-radiation equality affecting, potentially, CMB anisotropies [1]. Through the years, various studies have been devoted to the effect of large-scale magnetic fields on the vector and tensor CMB anisotropies [3] (see also [4] and references therein for some recent review articles).

The implications of fully inhomogeneous magnetic fields on the scalar modes of the geometry remain comparatively less explored. By fully inhomogeneous we mean stochastically distributed fields that do not break the spatial isotropy of the background [4]. One of the aims of the present paper is to partially bridge this gap and to open the way for further developments. In short the idea is the following. The simplest set of initial conditions for CMB anisotropies, implies, in a $\Lambda \mathrm{CDM}$ framework, that a nearly scale-invariant spectrum of adiabatic fluctuations is present after matter-radiation equality but before decoupling for typical wavelengths larger than the Hubble radius at the

*Electronic address: massimo.giovannini@cern.ch
PACS numbers: 98.70.Vc, 78.20.Ek, 98.62.En, 98.80.Cq

corresponding epoch [5]. It became relevant, through the years, to relax the assumption of exact adiabaticity and to scrutinize the implications of a more general mixture of adiabatic and nonadiabatic initial conditions (see [6] and references therein). In this paper it will be argued, along a similar perspective, that large-scale magnetic fields slightly modify the adiabatic paradigm so that their typical strengths may be constrained. To achieve such a goal, the first step is to solve the evolution equations of magnetized cosmological perturbations well before equality. The second step is to follow the solution through equality (and up to decoupling).

Consider then the system of cosmological perturbations of a flat Friedmann-Robertson-Walker (FRW) Universe, characterized by a conformal time scale factor $a(\tau)$, and consisting of a mixture of photons, baryons, CDM particles and massless neutrinos. In the conformally Newtonian gauge [6,7] the scalar fluctuations of the metric tensor $g_{\mu \nu}$ are parametrized in terms of the two longitudinal fluctuations i.e. $\delta g_{00}=2 a^{2} \phi(\tau, \vec{x}) \quad$ and $\quad \delta g_{i j}=$ $2 a^{2} \psi(\tau, \vec{x}) \delta_{i j}$. The Hamiltonian and momentum constraints, stemming from the (00) and $(0 i)$ components of the perturbed Einstein equations are:

$$
\begin{aligned}
\nabla^{2} \psi-3 \mathcal{H}\left(\mathcal{H} \phi+\psi^{\prime}\right) & =4 \pi G a^{2}\left[\delta \rho_{\mathrm{t}}+\delta \rho_{\mathrm{B}}\right], \\
\delta \rho_{\mathrm{B}}(\tau, \vec{x}) & =\frac{B^{2}(\vec{x})}{8 \pi a^{4}(\tau)}, \\
\nabla^{2}\left(\mathcal{H} \phi+\psi^{\prime}\right) & =-4 \pi G a^{2}\left(p_{\mathrm{t}}+\rho_{\mathrm{t}}\right) \theta_{\mathrm{t}}
\end{aligned}
$$

where $\mathcal{H}=a^{\prime} / a$ and the prime denotes a derivation with respect to the conformal time coordinate $\tau$. The total energy and pressure densities of the mixture, i.e. $\rho_{\mathrm{t}}=$ $\sum_{\mathrm{a}} \rho_{\mathrm{a}}$ and $p_{\mathrm{t}}=\sum_{\mathrm{a}} p_{\mathrm{a}}$, determine the evolution of the background geometry according to Friedmann equations:

$$
\begin{aligned}
\mathcal{H}^{2} & =\frac{8 \pi G}{3} a^{2} \rho_{\mathrm{t}}, \\
\mathcal{H}^{2}-\mathcal{H}^{\prime} & =4 \pi G a^{2}\left(\rho_{\mathrm{t}}+p_{\mathrm{t}}\right), \\
\rho_{\mathrm{t}}^{\prime}+3 \mathcal{H}\left(\rho_{\mathrm{t}}+p_{\mathrm{t}}\right) & =0 .
\end{aligned}
$$


In Eqs. (1) and (2) $\delta \rho_{\mathrm{t}}$ and $\theta_{\mathrm{t}}$, denote, respectively, the total density fluctuation of the fluid mixture and the divergence of the total velocity field (i.e. $\theta_{\mathrm{t}}=\partial_{i} v_{\mathrm{t}}^{i}$ ) whose expressions, in terms of the four components of the plasma, i.e. $\nu$, $\gamma, \mathrm{c}(\mathrm{CDM})$ and $\mathrm{b}$ (baryons), is

$$
\begin{aligned}
& \delta \rho_{\mathrm{t}}=\sum_{\mathrm{a}} \delta \rho_{\mathrm{a}}, \quad \delta p_{\mathrm{t}}=\sum_{\mathrm{a}} \delta p_{\mathrm{a}}, \\
& \left(p_{\mathrm{t}}+\rho_{\mathrm{t}}\right) \theta_{\mathrm{t}}=\sum_{\mathrm{a}}\left(p_{\mathrm{a}}+\rho_{\mathrm{a}}\right) \theta_{\mathrm{a}} .
\end{aligned}
$$

The spatial components of the perturbed Einstein equations, imply, instead

$$
\begin{aligned}
\psi^{\prime \prime}+ & \mathcal{H}\left(\phi^{\prime}+2 \psi^{\prime}\right)+\left(2 \mathcal{H}^{\prime}+\mathcal{H}^{2}\right) \phi+\frac{1}{3} \nabla^{2}(\phi-\psi) \\
= & 4 \pi G a^{2}\left(\delta p_{\mathrm{t}}+\delta p_{\mathrm{B}}\right) \\
\nabla^{4}(\phi-\psi) & =12 \pi G a^{2}\left[\left(p_{\nu}+\rho_{\nu}\right) \nabla^{2} \sigma_{\nu}+\left(p_{\gamma}+\rho_{\gamma}\right) \nabla^{2} \sigma_{\mathrm{B}}\right] \\
& \delta p_{\mathrm{B}}=\frac{\delta \rho_{\mathrm{B}}}{3} .
\end{aligned}
$$

In Eq. (6) $\nabla^{2} \sigma_{\nu}$ is the neutrino anisotropic stress, while $\nabla^{2} \sigma_{\mathrm{B}}$ is the magnetic field anisotropic stress defined as:

$$
\begin{aligned}
\nabla^{2} \sigma_{\mathrm{B}} & =\frac{3}{16 \pi a^{4} \rho_{\gamma}} \vec{\nabla} \cdot[(\vec{\nabla} \times \vec{B}) \times \vec{B}]+\frac{\nabla^{2} \Omega_{\mathrm{B}}}{4}, \\
\Omega_{\mathrm{B}}(\vec{x}) & =\frac{\delta \rho_{\mathrm{B}}(\tau, \vec{x})}{\rho_{\gamma}(\tau)},
\end{aligned}
$$

where, $\Omega_{\mathrm{B}}(\vec{x})$ is the magnetic energy density referred to the photon energy density and it is constant to a very good approximation if magnetic flux is frozen into the plasma element $[1,3,4]$, as assumed throughout the paper. The induced Ohmic current $\vec{J}$ is solenoidal (in the magnetohydrodynamical (MHD) description adopted here) and it is simply given by $4 \pi \vec{J}=\vec{\nabla} \times \vec{B}$. Moreover, in MHD, $\vec{E}=$ $\vec{J} / \sigma \simeq(\vec{\nabla} \times \vec{B}) / \sigma$ (where $\sigma$ is the conductivity). Since, prior to decoupling, the Universe was a rather good conductor [4], the contribution of the electric energy density and of the Poynting vector appearing, respectively, in Eqs. (1) and (2) can be safely neglected for typical length scales much larger than the screening length of the plasma. It should be stressed that, in Eq. (6), on top of the magnetic piece, the only contribution to the anisotropic stress of the fluid mixture comes from massless neutrinos ${ }^{1}$ (that are collisionless for temperatures smaller than $1 \mathrm{MeV}$ ) and it is parametrized by $\sigma_{\nu}$. The evolution of $\delta \rho_{\mathrm{t}}$ can be determined from the covariant conservation of the (total) energy-momentum tensor:

$$
\begin{aligned}
& \delta \rho_{\mathrm{t}}^{\prime}-3 \psi^{\prime}\left(p_{\mathrm{t}}+\rho_{\mathrm{t}}\right)+\left(p_{\mathrm{t}}+\rho_{\mathrm{t}}\right) \theta_{\mathrm{t}}+3 \mathcal{H}\left(1+c_{\mathrm{s}}^{2}\right) \delta \rho_{\mathrm{t}} \\
& \quad+3 \mathcal{H} \delta p_{\mathrm{nad}}=\frac{\vec{E} \cdot \vec{J}}{a^{4}}
\end{aligned}
$$

\footnotetext{
${ }^{1}$ If neutrinos would have a mass in the meV range, they would be nonrelativistic today but they will still be counted as radiation prior to decoupling.
}

where $c_{\mathrm{s}}^{2}=p_{\mathrm{t}}^{\prime} / \rho_{\mathrm{t}}^{\prime}$ is the (total) sound speed and where the (total) pressure density fluctuation $\delta p_{\mathrm{t}}$ has been slpit into the adiabatic contribution (i.e. $c_{\mathrm{s}}^{2} \delta \rho_{\mathrm{t}}$ ) supplemented by the nonadiabatic pressure density fluctuation (i.e. $\delta p_{\text {nad }}$ ). The electromagnetic contribution appearing in Eq. (8) contains an electric field and it is therefore suppressed. ${ }^{2}$ The evolution of the CDM component feels indirectly the presence of the magnetic field intensity through the Hamiltonian constraint (1) and the relevant equations are

$$
\theta_{\mathrm{c}}^{\prime}+\mathcal{H} \theta_{\mathrm{c}}+\nabla^{2} \phi=0, \quad \delta_{\mathrm{c}}^{\prime}=3 \psi^{\prime}-\theta_{\mathrm{c}}
$$

$\delta_{\mathrm{c}}$ is the CDM density contrast. The neutrinos are coupled to the magnetic field through the Hamiltonian constraint (1) and through Eq. (6) (involving the neutrino anisotropic stress $\left.\nabla^{2} \sigma_{\nu}\right)$ :

$$
\begin{gathered}
\theta_{\nu}^{\prime}+\frac{1}{4} \nabla^{2} \delta_{\nu}+\nabla^{2} \phi=\nabla^{2} \sigma_{\nu}, \quad \delta_{\nu}^{\prime}=4 \psi^{\prime}-\frac{4}{3} \theta_{\nu} \\
\sigma_{\nu}^{\prime}=\frac{4}{15} \theta_{\nu}
\end{gathered}
$$

where, in full analogy with Eq. (9) the neutrino density contrast $\delta_{\nu}$ has been introduced. Photons and baryons are tightly coupled by Thompson scattering and form, effectively, a single fluid characterized by a velocity field $\theta_{\gamma \mathrm{b}}=$ $\theta_{\gamma}=\theta_{\mathrm{b}}$. The relevant evolution equations are, in this case,

$$
\begin{aligned}
& \delta_{\gamma}^{\prime}=4 \psi^{\prime}-\frac{4}{3} \theta_{\gamma \mathrm{b}}, \quad \delta_{\mathrm{b}}^{\prime}=3 \psi^{\prime}-\theta_{\gamma \mathrm{b}}, \\
& R_{\mathrm{b}}(\tau)=\frac{3}{4} \frac{\rho_{\mathrm{b}}(\tau)}{\rho_{\gamma}(\tau)}=\left(\frac{698}{z+1}\right)\left(\frac{h^{2} \bar{\Omega}_{\mathrm{b}}}{0.023}\right), \\
& \theta_{\gamma \mathrm{b}}^{\prime}+\frac{\mathcal{H} R_{\mathrm{b}}}{\left(1+R_{\mathrm{b}}\right)} \theta_{\gamma \mathrm{b}}+\frac{\nabla^{2} \delta_{\gamma}}{4\left(1+R_{\mathrm{b}}\right)}+\nabla^{2} \phi \\
& \quad=\frac{3}{4} \frac{\vec{\nabla} \cdot[\vec{J} \times \vec{B}]}{a^{4} \rho_{\gamma}\left(1+R_{\mathrm{b}}\right)},
\end{aligned}
$$

where $R_{\mathrm{b}}$ is the baryon to photon ratio that depends on the redshift $z$. Deep in the radiation-dominated epoch i.e. for $\tau \ll \tau_{\text {eq }}$ the solution for the magnetized adiabatic mode can be obtained, in Fourier space, by solving, simultaneously, Eqs. (9)-(12). The compatibility of the magnetized adiabatic mode with Eqs. (1) and (2) and also with Eqs. (5) and (6) fixes the integration constants. Defining as $k$ the Fourier (comoving) wave-number we shall be interested in wavelengths much larger than the Hubble radius, i.e. $k \tau<1$. For $\tau \ll \tau_{\text {eq }}$, the density contrasts for the magnetized adiabatic mode are, to lowest order in $k \tau<1$,

\footnotetext{
${ }^{2}$ Nonlinear effects may arise in the MHD treatment of the problem. These effects may be connected with the presence (or absence) of Hall and thermoelectric terms in the effective Ohm law. Other nonlinear terms may contain two magnetic fields and the bulk velocity of the plasma. These terms are relevant when going to second order in the amplitude of the gravitational fluctuations but do not affect the results reported here.
} 


$$
\begin{aligned}
& \delta_{\gamma}=\delta_{\nu}=-2 \phi_{\mathrm{i}}-R_{\gamma} \Omega_{\mathrm{B}}, \\
& \delta_{\mathrm{b}}=\delta_{\mathrm{c}}=-\frac{3}{2} \phi_{\mathrm{i}}-\frac{3}{4} R_{\gamma} \Omega_{\mathrm{B}},
\end{aligned}
$$

$R_{\gamma}$ and $R_{\nu}$ are, respectively, the fractional contributions of photons and neutrinos to the radiation plasma. From Eqs. (9), (10), and (12) the velocity fields of the various species are

$$
\begin{gathered}
\theta_{\gamma \mathrm{b}}=\frac{k^{2} \tau}{4}\left[2 \phi_{\mathrm{i}}+R_{\nu} \Omega_{\mathrm{B}}-4 \sigma_{\mathrm{B}}\right], \quad \theta_{\mathrm{c}}=\frac{k^{2} \tau}{2} \phi_{\mathrm{i}}, \\
\theta_{\nu}=\frac{k^{2} \tau}{2}\left[\phi_{\mathrm{i}}-\frac{R_{\gamma} \Omega_{\mathrm{B}}}{2}\right]+k^{2} \tau \frac{R_{\gamma}}{R_{\nu}} \sigma_{\mathrm{B}} .
\end{gathered}
$$

The quantities $\psi_{\mathrm{i}}(k)$ and $\phi_{\mathrm{i}}(k)$ appearing in Eqs. (13) and (14) denote the super-Hubble fluctuations that are initially present prior to equality. By solving in terms of the neutrino anisotropic stress $\sigma_{\nu}$ and by recalling Eq. (6) the relation between $\psi_{\mathrm{i}}$ and $\phi_{\mathrm{i}}$ can be obtained:

$$
\begin{aligned}
\psi_{\mathrm{i}} & =\phi_{\mathrm{i}}\left(1+\frac{2}{5} R_{\nu}\right)+\frac{R_{\gamma}}{5}\left(4 \sigma_{\mathrm{B}}-R_{\nu} \Omega_{\mathrm{B}}\right), \\
\sigma_{\nu} & =-\frac{R_{\gamma}}{R_{\nu}} \sigma_{\mathrm{B}}+\frac{k^{2} \tau^{2}}{6 R_{\nu}}\left(\psi_{\mathrm{i}}-\phi_{\mathrm{i}}\right) .
\end{aligned}
$$

In the limit $\sigma_{\mathrm{B}} \rightarrow 0$ and $\Omega_{\mathrm{B}} \rightarrow 0$ this solution reproduces the standard adiabatic mode in the longitudinal gauge (see third and fourth references in [7]). To follow the fate of the magnetized adiabatic mode through $\tau_{\text {eq }}$ it is practical to exploit the total density contrast on uniform curvature hypersurfaces (conventionally denoted by $\zeta[7,8]$ ) or the curvature perturbation on comoving orthogonal hypersurfaces (conventionally denoted by $\mathcal{R}[7,8]$ ) whose specific definitions, in terms of the variables of the longitudinal gauge, are

$$
\begin{gathered}
\zeta=-\psi-\mathcal{H} \frac{\delta \rho_{\mathrm{t}}+\delta \rho_{\mathrm{B}}}{\rho_{\mathrm{t}}^{\prime}}, \\
\mathcal{R}=-\psi-\frac{\mathcal{H}\left(\mathcal{H} \phi+\psi^{\prime}\right)}{\mathcal{H} \mathcal{H}^{2}-\mathcal{H}^{\prime}}, \\
\zeta=\mathcal{R}+\frac{\nabla^{2} \psi}{12 \pi G a^{2}\left(p_{\mathrm{t}}+\rho_{\mathrm{t}}\right)} .
\end{gathered}
$$

The first and second relations in Eq. (16) are the definitions of $\zeta$ and $\mathcal{R}$ in terms of the conformally Newtonian variables. The third relation in Eq. (16) can be obtained by substituting the definitions of $\zeta$ and $\mathcal{R}$ back into Eq. (1) and by recalling the background relations (3). In the limit when the relevant wavelengths are all larger than the Hubble radius at the corresponding epoch, i.e. $k \tau<1$, the third relation in (16) implies that $\mathcal{R} \simeq \zeta$. The evolution equation for $\zeta$ can then be obtained by inserting the definition of $\zeta$ into Eq. (8); the result is

$$
\zeta^{\prime}=-\frac{\mathcal{H}}{p_{\mathrm{t}}+\rho_{\mathrm{t}}} \delta p_{\text {nad }}+\frac{\mathcal{H}}{p_{\mathrm{t}}+\rho_{\mathrm{t}}}\left(c_{\mathrm{s}}^{2}-\frac{1}{3}\right) \delta \rho_{\mathrm{B}}-\frac{\theta_{\mathrm{t}}}{3} .
$$

The nonadiabatic pressure density variation $\delta p_{\text {nad }}$ can be written as a sum of the relative entropy fluctuations over the various components of the mixture

$$
\begin{aligned}
& \delta p_{\mathrm{nad}}=\frac{1}{6 \mathcal{H} \rho_{\mathrm{t}}^{\prime}} \sum_{\mathrm{ij}} \rho_{\mathrm{i}}^{\prime} \rho_{\mathrm{j}}^{\prime}\left(c_{\mathrm{si}}^{2}-c_{\mathrm{sj}}^{2}\right) \mathcal{S}_{\mathrm{ij}}, \\
& \mathcal{S}_{i \mathrm{j}}=-3\left(\zeta_{\mathrm{i}}-\zeta_{\mathrm{j}}\right), \quad c_{\mathrm{si}}^{2}=\frac{p_{\mathrm{i}}^{\prime}}{\rho_{\mathrm{i}}^{\prime}},
\end{aligned}
$$

where $\mathcal{S}_{\mathrm{ij}}$ are the relative fluctuations in the entropy density that can be computed, from Eq. (18), in terms of the density contrasts of the individual fluids, i.e.

$$
\begin{gathered}
\zeta_{\mathrm{c}}=-\psi+\frac{\delta_{\mathrm{c}}}{3}, \quad \zeta_{\mathrm{b}}=-\psi+\frac{\delta_{\mathrm{b}}}{3}, \\
\zeta_{\nu}=-\psi+\frac{\delta_{\nu}}{4}, \quad \zeta_{\gamma}=-\psi+\frac{\delta_{\gamma}}{4}, \\
\zeta=\frac{\rho_{\nu}^{\prime} \zeta_{\nu}+\rho_{\gamma}^{\prime} \zeta_{\gamma}+\rho_{\mathrm{c}}^{\prime} \zeta_{\mathrm{c}}+\rho_{\mathrm{b}}^{\prime} \zeta_{\mathrm{b}}}{\rho_{\mathrm{t}}^{\prime}}+\zeta_{\mathrm{B}}, \\
\zeta_{\mathrm{B}}=\frac{\delta \rho_{\mathrm{B}}}{3\left(p_{\mathrm{t}}+\rho_{\mathrm{t}}\right)} .
\end{gathered}
$$

In the case of adiabatic (magnetized) initial conditions it can be easily verified that $\zeta_{\mathrm{c}}=\zeta_{\mathrm{b}}=\zeta_{\nu}=\zeta_{\gamma}$ so that $\delta p_{\text {nad }}=0$. Deep in the radiation-dominated epoch, for $\tau \ll \tau_{\text {eq }}, c_{\mathrm{s}}^{2} \rightarrow 1 / 3$ and, from Eq. (17), $\zeta^{\prime}=0$, so that

$$
\begin{aligned}
& \zeta=\zeta_{\mathrm{i}} \simeq \mathcal{R}_{\mathrm{i}}, \\
& \zeta_{\mathrm{i}}=-\frac{3}{2} \phi_{\mathrm{i}}\left(1+\frac{4}{15} R_{\nu}\right)-\frac{R_{\gamma}}{5}\left(4 \sigma_{\mathrm{B}}-R_{\nu} \Omega_{\mathrm{B}}\right) .
\end{aligned}
$$

When the Universe becomes matter-dominated, after $\tau_{\text {eq }}$, $c_{\mathrm{s}}^{2} \rightarrow 0$ and the second term at the right hand side of Eq. (17) does contribute significantly at decoupling (recall that for $h^{2} \Omega_{\text {matter }}=0.134, \tau_{\mathrm{dec}}=2.36 \tau_{\mathrm{eq}}$ ). Consequently, from Eq. (17), recalling that $c_{\mathrm{s}}^{2}=4 a_{\mathrm{eq}} /\left[3\left(3 a+4 a_{\mathrm{eq}}\right)\right]$, we obtain

$$
\zeta_{\mathrm{f}}=\zeta_{\mathrm{i}}-\frac{3 a R_{\gamma} \Omega_{\mathrm{B}}}{4\left(3 a+4 a_{\mathrm{eq}}\right)}, \quad \Omega_{\mathrm{Bf}}=\Omega_{\mathrm{Bi}} .
$$

The inclusion of one (or more) nonadiabatic modes changes the form of Eq. (17) and, consequently, the related solution (21). For instance, in the case of the CDMradiation nonadiabatic mode the relevant terms arising in the sum (18) are $\mathcal{S}_{\mathrm{c} \gamma}=\mathcal{S}_{\mathrm{c} \nu}=\mathcal{S}_{\mathrm{i}}$ where $\mathcal{S}_{i}$ is the (constant) fluctuation in the relative entropy density initially present (i.e. for $\tau \ll \tau_{\text {eq }}$ ). If this is the case $\delta p_{\text {nad }}=c_{\mathrm{s}}^{2} \rho_{\mathrm{c}} \mathcal{S}_{i}$ and Eq. (17) can be easily solved. The transfer matrix for magnetized $\mathrm{CMB}$ anisotropies can then be written as

$$
\left(\begin{array}{c}
\zeta_{\mathrm{f}} \\
\mathcal{S}_{\mathrm{f}} \\
\Omega_{\mathrm{Bf}}
\end{array}\right)=\left(\begin{array}{ccc}
\mathcal{M}_{\zeta \zeta} & \mathcal{M}_{\zeta \mathcal{S}} & \mathcal{M}_{\zeta \mathrm{B}} \\
0 & \mathcal{M}_{\mathcal{S S}} & \mathcal{M}_{\mathcal{S B}} \\
0 & 0 & \mathcal{M}_{\mathrm{BB}}
\end{array}\right)\left(\begin{array}{c}
\zeta_{\mathrm{i}} \\
\mathcal{S}_{\mathrm{i}} \\
\Omega_{\mathrm{Bi}}
\end{array}\right) .
$$

In the case of a mixture of (magnetized) adiabatic and CDM-radiation modes, we find, for $a>a_{\mathrm{eq}}$

$$
\begin{gathered}
\mathcal{M}_{\zeta \zeta} \rightarrow 1, \quad \mathcal{M}_{\zeta \mathcal{S}} \rightarrow-\frac{1}{3}, \quad \mathcal{M}_{\zeta \mathrm{B}}-\frac{R_{\gamma}}{4}, \\
\mathcal{M}_{\mathcal{S S}} \rightarrow 1, \quad \mathcal{M}_{\mathcal{S B}} \rightarrow 0,
\end{gathered}
$$


and $\mathcal{M}_{\mathrm{BB}} \rightarrow 1$. Eqs. (22) and (23) may be used, for instance, to obtain the magnetized curvature and entropy fluctuations at photon decoupling in terms of the same quantities evaluated for $\tau \ll \tau_{\text {eq }}$. A full numerical analysis of the problem confirms the analytical results summarized by Eqs. (22) and (23). The most general initial condition for CMB anisotropies will then be a combination of (correlated) fluctuations receiving contribution from $\delta p_{\text {nad }}$ and from the fully inhomogeneous magnetic field. To illustrate this point, the form of the Sachs-Wolfe (SW) plateau in the sudden decoupling limit will now be discussed.

$$
\begin{aligned}
\delta_{\gamma}^{\prime \prime} & +\frac{\mathcal{H} R_{\mathrm{b}}}{1+R_{\mathrm{b}}} \delta_{\gamma}^{\prime}+\frac{k^{2}}{3} \frac{\delta_{\gamma}}{1+R_{\mathrm{b}}} \\
& =4 \psi^{\prime \prime}+\frac{4 \mathcal{H} R_{\mathrm{b}}}{1+R_{\mathrm{b}}} \psi^{\prime}-\frac{4}{3} k^{2} \phi-\frac{k^{2}}{3\left(1+R_{\mathrm{b}}\right)}\left(\Omega_{\mathrm{B}}-4 \sigma_{\mathrm{B}}\right) .
\end{aligned}
$$

In the sudden decoupling approximation the visibility function, i.e. $\mathcal{K}(\tau)=\kappa^{\prime}(\tau) e^{-\kappa(\tau)}$ and the optical depth, i.e. $e^{-\kappa(\tau)}$ are approximated, respectively, by $\delta\left(\tau-\tau_{\mathrm{dec}}\right)$ and by $\theta\left(\tau-\tau_{\mathrm{dec}}\right)$. The power spectra of $\zeta, \mathcal{S}$ and $\Omega_{\mathrm{B}}$ are

$$
\begin{gathered}
\mathcal{P}_{\zeta}(k)=\mathcal{A}_{\zeta}\left(\frac{k}{k_{\mathrm{p}}}\right)^{n_{r}-1}, \quad \mathcal{P}_{\mathcal{S}}(k)=\mathcal{A}_{\mathcal{S}}\left(\frac{k}{k_{\mathrm{p}}}\right)^{n_{s}-1}, \\
\mathcal{P}_{\Omega}(k)=\mathcal{F}(\varepsilon) \bar{\Omega}_{\mathrm{B} L}^{2}\left(\frac{k}{k_{L}}\right)^{2 \varepsilon},
\end{gathered}
$$

where $\mathcal{A}_{\zeta}, \mathcal{A}_{\mathcal{S}}$ and $\bar{\Omega}_{\mathrm{B} L}$ are constants and

$$
\begin{gathered}
\mathcal{F}(\varepsilon)=\frac{4(6-\varepsilon)(2 \pi)^{2 \varepsilon}}{\varepsilon(3-2 \varepsilon) \Gamma^{2}(\varepsilon / 2)}, \quad \bar{\Omega}_{\mathrm{B} L}=\frac{\rho_{\mathrm{B} L}}{\bar{\rho}_{\gamma}}, \\
\rho_{\mathrm{B} L}=\frac{B_{L}^{2}}{8 \pi}, \quad \bar{\rho}_{\gamma}=a^{4}(\tau) \rho_{\gamma}(\tau) .
\end{gathered}
$$

To deduce Eqs. (25) and (26) the magnetic field has been regularized, according to a common practice $[3,4]$, over a typical comoving scale $L=2 \pi / k_{L}$ with a Gaussian window function and it has been assumed that the magnetic field intensity is stochastically distributed as

$$
\left\langle B_{i}(\vec{k}, \tau) B^{j}(\vec{p}, \tau)\right\rangle=\frac{2 \pi^{2}}{k^{3}} P_{i}^{j}(k) P_{\mathrm{B}}(k, \tau) \delta^{(3)}(\vec{k}+\vec{p}),
$$

where $^{3}$

$$
P_{i}^{j}(k)=\left(\delta_{i}^{j}-\frac{k_{i} k^{j}}{k^{2}}\right), \quad P_{\mathrm{B}}(k, \tau)=A_{\mathrm{B}}\left(\frac{k}{k_{\mathrm{p}}}\right)^{\varepsilon} .
$$

As a consequence of Eq. (27) the magnetic field does not

\footnotetext{
${ }^{3}$ Different conventions can be adopted for the magnetic power spectrum $[3,4]$. The conventions followed here differ slightly from the ones employed by other authors, since, in the present case, the form of the magnetic correlator has to be consistent with the form of the power spectrum of curvature and entropy perturbations. In particular, the magnetic spectral index defined as $n$ in [3] (fourth and fifth references) correspond, in the present notations, to $\varepsilon-3$. When magnetic fields are fully inhomogeneous but helical a second (antisymmetric) term arises in Eq. (27) (see [3], last reference). This term does not affect the discussion of the scalar modes.
}

break the spatial isotropy of the background geometry. The quantity $k_{\mathrm{p}}$ appearing in Eqs. (25) and (28) is conventional pivot scale that is $0.05 \mathrm{Mpc}$ (see [6] for a discussion of other possible choices). Eqs. (25) and (26) hold for $0<$ $\varepsilon<1$. In this limit the $\mathcal{P}_{\Omega}(k)$ (see Eq. (25)) is nearly scaleinvariant (but slightly blue). This means that the effect of the magnetic and thermal diffusivity scales (related, respectively, to the finite value of the conductivity and of the thermal diffusivity coefficient) do not affect the spectrum [4]. In the opposite limit, i.e. $\varepsilon \gg 1$ the value of the modecoupling integral appearing in the two-point function of the magnetic energy density (and of the magnetic anisotropic stress) is dominated by ultraviolet effects related to the mentioned diffusivity scales [4]. Using then Eqs. (25) and (26) the $C_{\ell}$ can be computed for the region of the SW plateau (i.e. for multipoles $\ell<30$ ):

$$
\begin{aligned}
C_{\ell}= & {\left[\frac{\mathcal{A}_{\zeta}}{25} Z_{1}\left(n_{r}, \ell\right)+\frac{9}{100} R_{\gamma}^{2} \bar{\Omega}_{\mathrm{B} L}^{2} Z_{2}(\epsilon, \ell)\right.} \\
& -\frac{4}{25} \sqrt{\mathcal{A}_{\zeta} \mathcal{A}_{\mathcal{S}}} Z_{1}\left(n_{r s}, \ell\right) \cos \gamma_{r s}+\frac{4}{25} \mathcal{A}_{\mathcal{S}} Z_{1}\left(n_{s}, \ell\right) \\
& -\frac{3}{25} \sqrt{\mathcal{A}_{\zeta}} R_{\gamma} \bar{\Omega}_{\mathrm{B} L} Z_{3}\left(n_{r}, \varepsilon, \ell\right) \cos \gamma_{b r} \\
& \left.+\frac{6}{25} \sqrt{\mathcal{A}_{\mathcal{S}}} R_{\gamma} \bar{\Omega}_{\mathrm{B} L} Z_{3}\left(n_{s}, \varepsilon, \ell\right) \cos \gamma_{b s}\right]
\end{aligned}
$$

where the functions $Z_{1}, Z_{2}$ and $Z_{3}$

$$
\begin{aligned}
Z_{1}(n, \ell)= & \frac{\pi^{2}}{4}\left(\frac{k_{0}}{k_{\mathrm{p}}}\right)^{n-1} 2^{n} \frac{\Gamma(3-n) \Gamma\left(\ell+\frac{n-1}{2}\right)}{\Gamma^{2}\left(2-\frac{n}{2}\right) \Gamma\left(\ell+\frac{5}{2}-\frac{n}{2}\right)} \\
Z_{2}(\varepsilon, \ell)= & \frac{\pi^{2}}{2} 2^{2 \varepsilon} \mathcal{F}(\varepsilon)\left(\frac{k_{0}}{k_{L}}\right)^{2 \varepsilon} \frac{\Gamma(2-2 \varepsilon) \Gamma(\ell+\varepsilon)}{\Gamma^{2}\left(\frac{3}{2}-\varepsilon\right) \Gamma(\ell+2-\varepsilon)} \\
Z_{3}(n, \varepsilon, \ell)= & \frac{\pi^{2}}{4} 2^{\varepsilon} 2^{(n+1) / 2} \sqrt{\mathcal{F}(\varepsilon)}\left(\frac{k_{0}}{k_{L}}\right)^{\varepsilon}\left(\frac{k_{0}}{k_{\mathrm{p}}}\right)^{(n+1) / 2} \\
& \times \frac{\Gamma\left(\frac{5}{2}-\varepsilon-\frac{n}{2}\right) \Gamma\left(\ell+\frac{\varepsilon}{2}+\frac{n}{4}-\frac{1}{4}\right)}{\Gamma^{2}\left(\frac{7}{4}-\frac{\varepsilon}{2}-\frac{n}{4}\right) \Gamma\left(\frac{9}{4}+\ell-\frac{\varepsilon}{2}-\frac{n}{4}\right)}
\end{aligned}
$$

are defined in terms of the magnetic tilt $\varepsilon$ and of a generic spectral index $n$ which may correspond, depending on the specific contribution, either to $n_{r}$ (adiabatic spectral index), or to $n_{s}$ (nonadiabatic spectral index) or even to $n_{r s}=$ $\left(n_{r}+n_{s}\right) / 2$ (spectral index of the cross-correlation). In Eq. (29) $\gamma_{r s}, \gamma_{b r}$ and $\gamma_{s b}$ are the correlation angles. In the absence of magnetic and nonadiabatic contributions and for Eqs. (29) and (30) imply that for $n_{r}=1$ (Harrison-Zeldovich spectrum) $\quad \ell(\ell+1) C_{\ell} / 2 \pi=$ $\mathcal{A}_{\zeta} / 25$ and WMAP data [5] would imply that $\mathcal{A}_{\zeta}=$ $2.65 \times 10^{-9}$. Consider then the physical situation where on top of the adiabatic mode there is a magnetic contribution. If there is no correlation between the magnetized contribution and the adiabatic contribution, i.e. $\gamma_{b r}=$ $\pi / 2$, the SW plateau will be enhanced in comparison with the case when magnetic fields are absent. The same situation arises when the two components are anticorre- 
lated (i.e. $\cos \gamma_{b r}<0$ ). However, if the fluctuations are positively correlated (i.e. $\cos \gamma_{b r}>0$ ) the cross-correlation adds negatively to the sum of the two autocorrelations of $\zeta$ and $\Omega_{\mathrm{B}}$ so that the total result may be an overall reduction of the power with respect to the case $\gamma_{b r}=\pi / 2$. In Eq. (30)-(32) $k_{0}=\tau_{0}^{-1}$ where $\tau_{0}$ is the present observation time. Taking as typical parameters $h^{2} \Omega_{\mathrm{c}}=0.111, h^{2} \Omega_{\mathrm{b}}=$ 0.023 and $h^{2} \Omega_{\mathrm{r}}=4.15 \times 10^{-5}$ (with $h=0.73$ ), the amplitude of the magnetic field intensity can be constrained by requiring that the adiabatic mode dominates. In the case when the magnetic and adiabatic contribution are totally anticorrelated (i.e. $\cos \gamma_{b r}=-1$ ), which is the most unfavourable case the bound on the protogalactic field read, at the present epoch and over a typical comoving scale $L=$ $2 \pi / k_{L}$ with $k_{L}=\mathrm{Mpc}^{-1}$

$$
B_{L}<2.5 \times 10^{-9} \mathrm{G}, \quad n_{r} \simeq 0.951, \quad \varepsilon \simeq 0.9 .
$$

As indicated, the bound (33) assumes a nearly scaleinvariant (but slightly red [5]) adiabatic mode and the maximally allowed magnetic spectral tilt. A further reduction of $\varepsilon$ leads to a slight relaxation of the bound; for instance for $\varepsilon \simeq 0.4, B_{L}<6.3 \times 10^{-9} \mathrm{G}$. The bounds obtained in the present discussion are compatible with the ones based on different physical considerations (see Ref. [9] and references therein). In particular two independent groups (first and second references of [9]) have successfully attempted to constrain cosmological Alfvén turbulence from WMAP-1 data by looking at the correlation of the $a_{\ell-1, m}$ and $a_{\ell+1, m}$ multipole coefficients arising in the angular power spectrum as a result of a slight breaking of spatial isotropy. In the second paper of [9] possible non-Gaussian signals, arising in this framework, have been analyzed in the light of WMAP-1 data. The magnetic field, in the present case, is fully inhomogeneous and stochastic so that the spatial isotropy is unbroken. It is also worth mentioning that primordial magnetic fields can be generated in a variety of mechanisms in the early Universe [1,4]. For instance, fully inhomogeneous magnetic fields relevant for the present analysis can be produced in the case of Ref. [10].

In CMB physics is common practice to perform modelindependent analysis on the parameter space of the allowed initial conditions by including, for instance, correlated (or anticorrelated) nonadiabatic modes in the game (see, for instance, [6] and references therein). Up to now the effects related to fully inhomogeneous magnetic fields have been discussed within a different standard whose limitations were the impossibility of defining accurately both initial conditions and normalization of the magnetized (scalar) CMB anisotropies. The results reported here allow to overcome these difficulties and lead naturally to a strategy of parameter extraction where large-scale magnetic fields are treated consistently as a further degree of freedom in the space of the initial conditions. According to this perspective, it will be important to pursue the analysis of smallscale effects by semianalytical methods to corroborate and interpret more numerical studies related to parameter extraction.
[1] F. Govoni and L. Feretti, Int. J. Mod. Phys. D 13, 1549 (2004); B. M. Gaensler, R. Beck, and L. Feretti, New Astron. Rev. 48, 1003 (2004); R. Cassano, G. Brunetti, and G. Setti, astro-ph/0601702; Y. Xu, P. P. Kronberg, S. Habib, and Q. W. Dufton, Astrophys. J. 637, 19 (2006); M. Giovannini, Int. J. Mod. Phys. D 13, 391 (2004).

[2] A. Brandenburg and K. Subramanian, Phys. Rep. 417, 1 (2005); A. Lazarian, E. Vishniac, and J. Cho, Astrophys. J. 603, 180 (2004); Lect. Notes Phys. 614, 376 (2003).

[3] J. Barrow and K. Subramanian, Phys. Rev. Lett. 81, 3575 (1998); Phys. Rev. D 58, 103502 (1998); C. Tsagas and R. Maartens, Phys. Rev. D 61, 083519 (2000); A. Mack, T. Kahniashvili, and A. Kosowsky, Phys. Rev. D 65, 123004 (2002); A. Lewis, Phys. Rev. D 70, 043518 (2004); T. Kahniashvili and B. Ratra, Phys. Rev. D 71, 103006 (2005).

[4] K. Subramanian, astro-ph/0601570; M. Giovannini, Class. Quant. Grav. 23, R1 (2006).
[5] H. V. Peiris et al. (WMAP Collaboration), Astrophys. J. Suppl. Ser. 148, 213 (2003); D. Spergel et al. (WMAP Collaboration), astro-ph/0603449.

[6] K. Enqvist, H. Kurki-Suonio, and J. Valiviita, Phys. Rev. D 62, 103003 (2000); H. Kurki-Suonio, V. Muhonen, and J. Valiviita, Phys. Rev. D 71, 063005 (2005).

[7] E. Harrison, Rev. Mod. Phys. 39, 862 (1967); J. M. Bardeen, Phys. Rev. D 22, 1882 (1980); C.-P. Ma and E. Bertschinger, Astrophys. J. 455, 7 (1995); M. Giovannini, Phys. Rev. D 70, 123507 (2004).

[8] J. Bardeen, P. Steinhardt, and M. Turner, Phys. Rev. D 28, 679 (1983); R. Brandenberger, R. Kahn, and W. Press, Phys. Rev. D 28, 1809 (1983).

[9] G. Chen et al., Astrophys. J. 611, 655 (2004); P. D. Naselsky et al., ibid. 615, 45 (2004); D. G. Yamazaki et al., ibid. 625, L1 (2005).

[10] B. Ratra, Astrophys. J. 391, L1 (1992); see also [1,4] for other models leading to large-scale magnetic fields relevant for this analysis. 